\title{
Calculus: Eight Minutes by Vernacular-Fewer but Better
}

\author{
Qun Lin \\ Chinese Academy of Sciences, Beijing, China
}

Calculus, including differentials, integral and their relationship, is transferred to arithmetic level

Hypothesis: each $1>\frac{\text { numerator }}{\text { deno } \min \text { ator }}>0.9 \cdots 9 \Rightarrow$ Theorem: $1>\frac{\text { sum of numerators }}{\text { sum of denominators }}>0.9 \cdots 9$

and then is proved by a few lines of arithmetic, where

either $\quad$ numerator $=$ differential $($ tangent height), and denominator $=$ sub-height of the curve

or numerator $=$ sub-height of the curve, and denominator $=$ differential (tangent height $)$

For more details and a simple result, see the conclusion at the end of the paper.

Keywords: calculus, differential, arithmetic

\section{Introuduction}

The paper proposes that "One course, one theorem, explanation with a number $0 . \dot{9}$, proof by vernacular in 8 minutes," and the rest of it only plays such a theorem.

Previous example: A geometry, a parallel line axiom (see Figure 1).

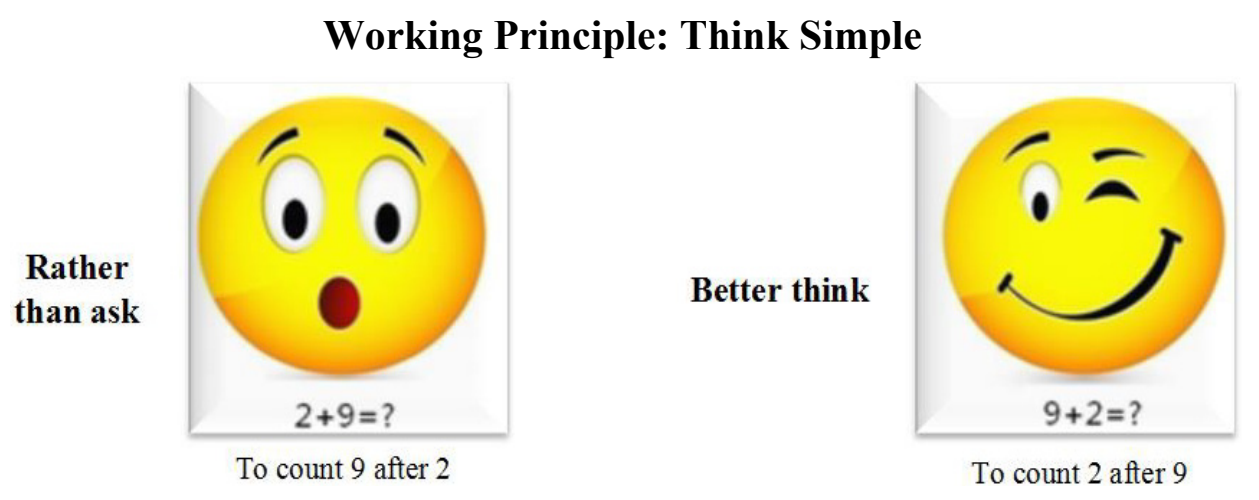

Figure 1. Children's arithmetic.

\section{What Teach in Lesson One: A Common Theorem/or Common Platform?}

Nowadays, numbers are frequently adopted in many TV programs, such as "News in numbers," "Daily Talk in numbers," eight minutes as a limit is use to control various speeches. Thus, they must be "fewer but better."

However, there were Leonardo da Vinci's "Painting in a percentage, 0.618" and Luogeng Hua's (1981) "Optimization in a percentage, 0.618 ," even further "fewer but better."

\footnotetext{
"Acknowledgements: The author would like to thank his colleagues, Xiaohuan Xue, Chunguang You, and Aijun Zhang, for their valuable help.

Qun Lin, professor and member of Chinese Academy of Sciences, Institute of Computational Mathematics and Scientific/Engineering Computing, Chinese Academy of Sciences.
} 
Can we, corresondently, have "Calculus in a percentage, $0 . \dot{9}$ ?"

Good news (a little exaggerates): Calculus can even be re-organized under a "common theorem."

$$
\int_{a}^{b} f^{\prime}(x) d x=f(b)-f(a) \text { (Newton Leibniz formula) }
$$

and the rest of it plays only the trick on such a "common theorem," i.e., one theorem wins one calculus world (see Figure 2).

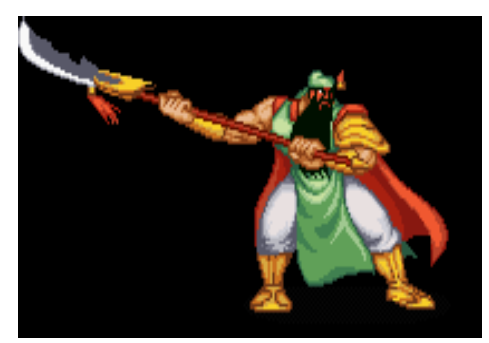

Figure 2. Kuan Kung, a famous general in Chinese history, plays a broadsword in his whole life.

Kuan Kung, a famous general in Chinese history, plays a broadsword in his whole life.

Bad news (a little exaggerates): The cost of itself, unfortunately, is too high: The explanation and proof for this theorem, in the traditional textbooks, even requires 80 hours' lessons, which to most people is already a high threshold.

Let us forget the traditional textbooks, but simply think calculus as the "Comics in a percentage, $0 . \dot{9}$. "

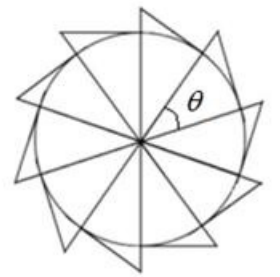

Circumference

$\frac{\text { Arc length }}{\text { Tangent length }}=0.999 \ldots$

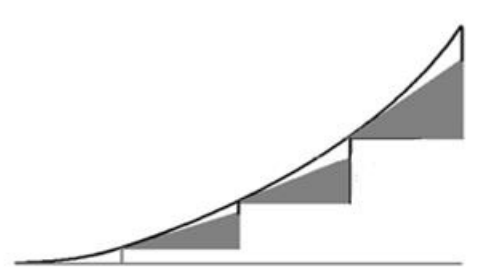

Curve length

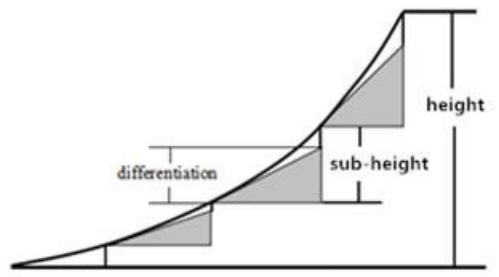

Curve height

$$
\frac{\text { Tangent length }}{\text { Sub }- \text { curve length }}=0.999 \ldots
$$

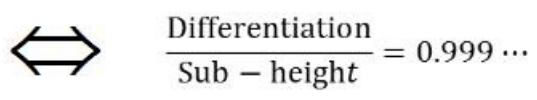

Figure 3. Calculus comics.

The three pictures have a common point: The numerator is so close to the denominator that the ratio reaches $0 . \dot{9}(=1)$. We can deduct, as a deduction from a simple lemma, that the ratio of the sum of the numerators to the sum of the denominators reaches $0 . \dot{9} \quad(=1)$ as well.

$$
\frac{\text { Circumference }}{\text { Sum of tangent lengths }}=0.999 \ldots
$$

$$
\begin{aligned}
& \frac{\text { Sum of tangent lengths }}{\text { Total curve length }}=0.999 \ldots \\
& \frac{\text { Sum of differentiations }}{\text { Total height }}=0.999 \ldots
\end{aligned}
$$


where, differentiation $=$ tangent height. So, we may define:

$$
\begin{aligned}
& \text { Integral of tangent length }=\text { Curve length } \\
& \text { Integral of differentiations }=\text { Total height }
\end{aligned}
$$

Different points: The right hand side of former formula is the length of a curve, while the right hand side of latter formula is the length of a straight line (height). Last formula, under the mathematical symbols, is just the Newton-Leibniz formula, which is called the "common theorem," or what said in Tolstoy's War and Peace (see Figure 4).

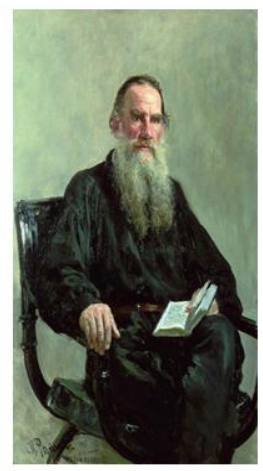

$$
\begin{aligned}
& \text { "Only by taking infinitesimally small } \\
& \text { units for observation (the differential of } \\
& \text { history, that is, the individual tendencies } \\
& \text { of men) and attaining to the art of } \\
& \text { integrating them (that is, finding the sum } \\
& \text { of these infinitesimals) can we hope to } \\
& \text { arrive at the laws of history." }
\end{aligned}
$$

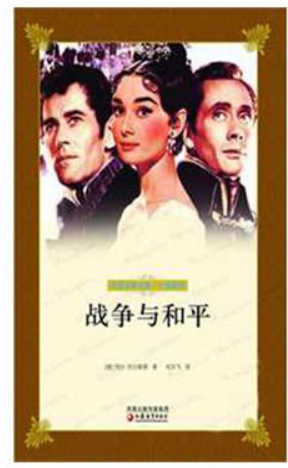

Figure 4. Writer and the cover of War and Peace.

We now go to prove the simple lemma, which uses a few lines of arithmetic by vernacular: Lemma, no matter what the numerator or denominator is, we will have:

Case 1: When sum of numerator $<$ sum of denominator, we have

$$
\text { each } \underset{\text { denominator }}{\text { numerator }}>0.9 \cdots 9 \Rightarrow \frac{\text { sum of numerator }}{\text { sum of denominator }}>0.9 \cdots 9
$$

Proof: Since numerator $>0.9 \cdots 9$ denominator, we have

$$
\text { sum of numerator }>0.9 \cdots 9 \text { sum of denominator }
$$

where any number of 9's can be added.

Case 2, since we did not define what are the numerator and denominator, they can be replaced by the denominator and numerator, respectively:

When sum of denominator<sum of numerator, we have:

$$
\text { each } \frac{\text { denominator }}{\text { numerator }}>0.9 \cdots 9 \Rightarrow \frac{\text { sum of denominator }}{\text { sum of numerator }}>0.9 \cdots 9
$$

where any number of 9's can be added.

Case 1 applies to the increasing and convex curves (which happens when calculating the circumference) (see Figure 5), where,

$$
\text { numerator }=\text { differentiation }<\text { denominator }=\text { sub-height }
$$




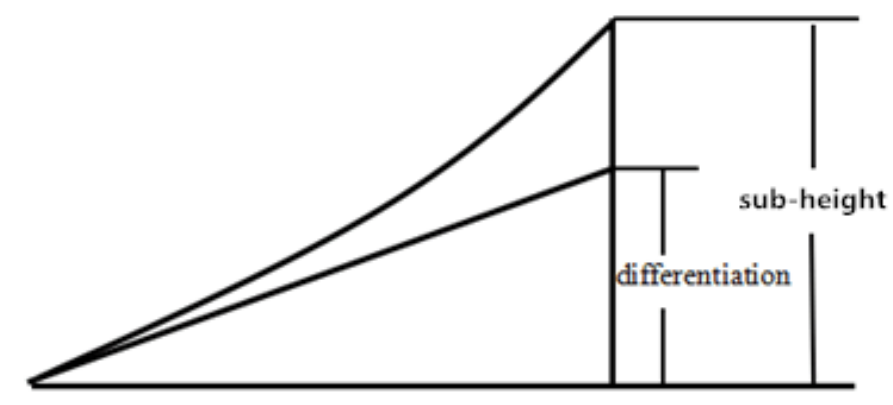

Figure 5. Convex curve.

and so is their sums. Thus, by $0 . \dot{9}(=1)$, we obtain at once the "common theorem":

$$
\text { Integral of differentiation }=\text { Total height }
$$

Case 2 applies to the increasing and concave curves (e.g., when calculating the circular area) (see Figure $6)$.

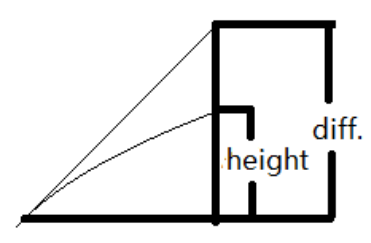

Figure 6. Concave curve.

For the non-convex, non-concave but differentiable curves, we have either the case 1 or the case 2 , but the two conditions under the two cases hold simultaneously at each point (see Appendix).

$$
\begin{gathered}
\frac{1}{0.9 \cdots 9}>\frac{\text { differentials }}{\text { sub-heights }}>0.9 \cdots 9 \\
\Rightarrow \frac{1}{0.9 \cdots 9}>\frac{\text { sum of differentials }}{\text { sum of sub-heights }}>0.9 \cdots 9
\end{gathered}
$$

Thus,

$$
\text { either } 1>\frac{\text { sum of differentiations }}{\text { total height }}>0.9 \cdots 9 \text { or } 1>\frac{\text { total height }}{\text { sum of differentiations }}>0.9 \cdots 9
$$

we still have the "common theorem."

An elementary proof of the "common theorem" is completed now, where we used a few painting (without coordinate) and a few lines of arithmetic (with no skills), a total of eight minutes of vernacular (without terminologies). So, ordinary people can do.

The threshold of the "common theorem" is greatly reduced now, fewer but better.

\section{What Will Be Taught in the Next Lessons?}

The "common theorem" is number one in the calculus course, since it is the common platform. And so, it must be taught in Lesson one. The future lessons only play the "common theorem." They are of different levels for 
different majors and different people. For example, besides the "common theorem," the humanities might be contented with other few lessons, like Lessons 2-5. Sciences, however, need more, like Lessons 6-10. And, many of them can be considered as discussions or exercises, completed in the classroom by teachers and students.

Then, calculus is a flexible teaching material or life education.

In short, past teaching materials for calculus should be re-organized under the "common theorem." One theorem is indeed enough — do not make more theorems to add mess.

In this paper, we only give Lesson 2 to strengthen Lesson 1, and an appendix to strengthen the lemma, but put other lessons in the Chinese versions (see Figure 7).

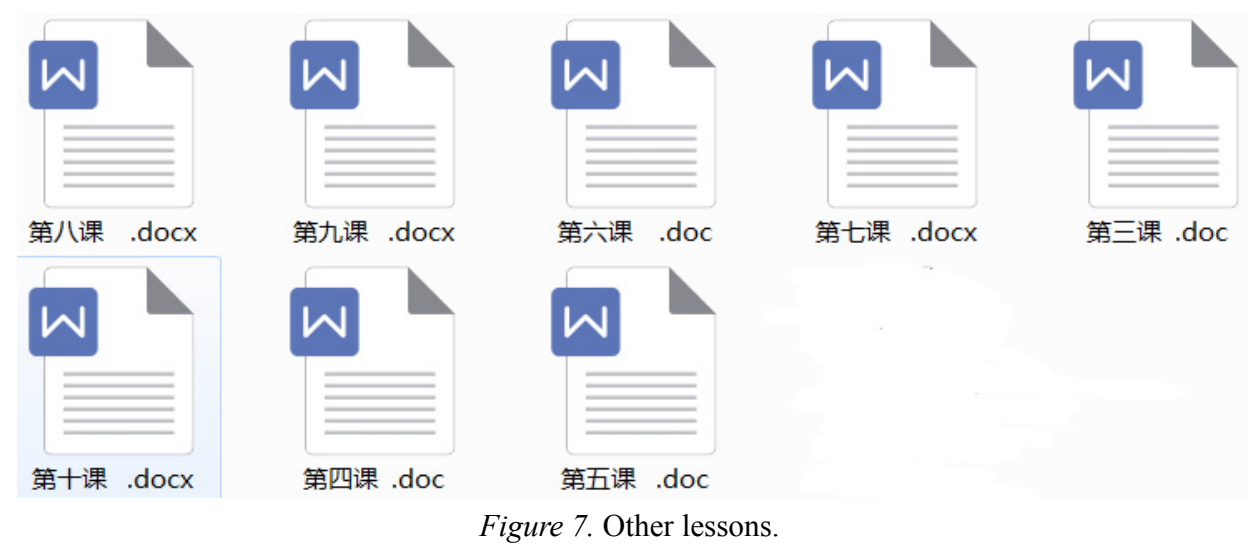

We have to admit, however, that the "calculus water" is very deep: The depth of calculus does not see the bottom. For example, how to give the multivariate calculus? And, Johnson's book Body and Soul proposes many unsolved problems. Hence, knowledge has no limit.

\section{Lesson Two: Three Comics in Calculus}

\section{Zhuangzi's Saying in Numbers}

Great oaks from little acorns grow-calculus based on the concept of infinite (see Figure 8).

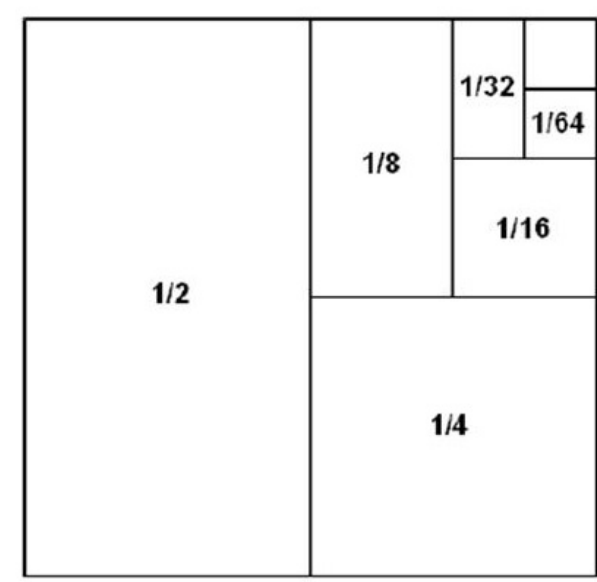

https://en. wikipedia. org/wiki/1/2_\%2B _1/4_\%2B_1/8_\%2B_1/16_\%2B_.

\section{Zhuangzi ( $\sim 300$ B.C.):}

Take half from a foot long stick each day, you will never exhaust it in million years.

Tear $1 \times 1$ paper into $1 / 2,1 / 4,1 / 8,1 / 16, \cdots$ pieces.

Put together all the pieces,

$$
\frac{1}{2}+\frac{1}{4}+\frac{1}{8}+\frac{1}{16}+\cdots=1
$$

This process brings about the infinite concept and infinite arithmetic.

Stepping from finite to infinite is a giant leap!

Figure 8. Sketch map of Zhuangzi's philosophy. 
The process in numbers: Collect the data (see Figure 9).

\begin{tabular}{|c|ll|}
\hline \#Terms & \multicolumn{2}{|c|}{ Sum } \\
\hline 4 & 0.9375 & (one 9) \\
7 & 0.9921875 & (two 9's) \\
10 & 0.9990234375 & (three 9's) \\
14 & 0.99993896484375 & (four 9's) \\
17 & 0.99999237060546875 & (five 9's) \\
$\ldots \ldots$ & $\ldots \ldots .$. & \\
34 & $0.99999999994179233 \ldots$ & (ten 9's)
\end{tabular}

Figure 9. Data table.

PS: Gold's purity is also through the numbers, the percentage of 9, 99, 999, and 9999. They are incredibly similar!

Concern: Would the process of adding 9 stop after a certain number of 9's?

Answer: No! If 9 does not keep being added, it could not reach 1. It must have infinite numbers of 9's (see Figure 10).

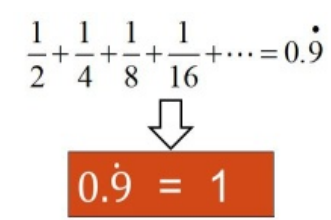

Figure 10. Proved by data.

The Zhuangzi's principle can be a prerequisite for learning calculus. Next, as we enter the core part of calculus, the trilogy of calculus .

\section{The First Play: Circle and Polygon}

Calculus originates from measuring the circumference.

Length could be measured by a ruler. How about the curve?

The circle drawn by computer, if magnified, becomes a regular polygon.
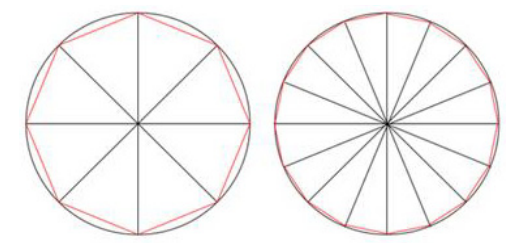

Secant line approximation

(Lower approximate)

Figure 11. Cyclotomic method.

This is the cyclotomic method of Archimedes and LIU Hui (see Figure 11).

Observation: The chord length and the arc length get closer and closer.

What is the meaning of close? It is also explained in numbers: Collect the data (see Figure 12).

Infinite number of 9's will be added, as $0 . \dot{9}(=1)$ or numerator = denominator. Define:

Integral of chord lengths $=$ Circumference 
An important step: Simplify the secant line to tangent line (Only related to a single tangent point) (see Figure 13).

\begin{tabular}{|c|c|c|}
\hline N-Polygon & Perimeter & Percentage \\
\hline 6 & 6 & 0.9549296586 \\
\hline 7 & 6.0743723476 & 0.9667663853 \\
\hline 8 & 6.1229349178 & 0.9744953584 \\
\hline 12 & 6.2116570824 & 0.9886159295 \\
\hline 14 & 6.2305861508 & 0.9916285843 \\
\hline 16 & 6.2428903046 & 0.9935868512 \\
\hline
\end{tabular}

\section{Percentage:}

$$
\frac{\text { chord length }}{\text { arc length }}\left(=\frac{\sin \frac{\theta}{2}}{\frac{\theta}{2}}\right)=0.999 \cdots \rightarrow \frac{\text { Sum of chord lengths }}{\text { Circumference }}=0.999 \ldots
$$

Figure 12. Proved by trigonometry.

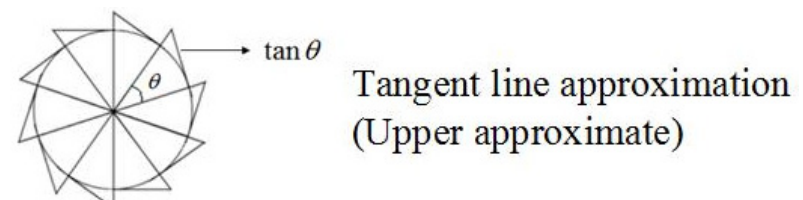

Figure 13. Cyclotomic method.

Observation: The tangent length and the arc length get closer and closer (see Figure 14).

By similar process, collect numbers, then observe the percentage,

$$
\begin{gathered}
\frac{\text { Arc length }}{\text { Tangent length }}\left(=\frac{\theta}{\tan \theta}\right)=0.999 \cdots \rightarrow \frac{\text { Circumference }}{\text { Sum of tagent lengths }}=0.999 \cdots \\
\text { Figure 14. Proved by trigonometry. }
\end{gathered}
$$

Infinite number of 9's will be added, as $0 . \dot{9}(=1)$ or denominator $=$ numerator. Define

$$
\text { Integral of tangent lengths }=\text { Circumference }
$$

Bad news: The above definitions cannot give the exact value, but always the approximate value. This has been a historical puzzle.

\section{The Second Plays : Curved Triangle and Tangent}

Let us extend from the circle to the general curve (e.g., hillside).

The curve length is unmeasurable. We may change it to straight line.

The curved triangle can be changed to straight triangle, e.g., the tangent triangle with hypotenuse tangent to the curve (see Figure 15).

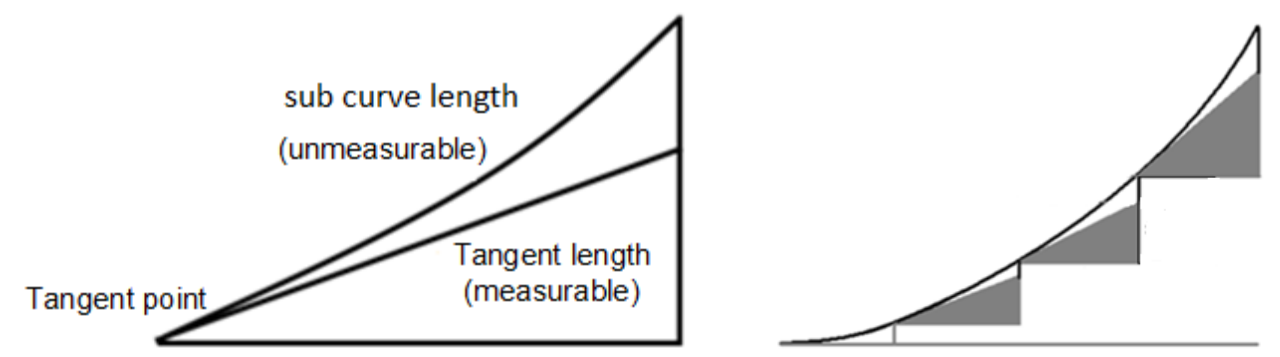

Figure 15. Sub-triangle and global triangle. 
Similar as last lecture about circle's tangent (i.e., collect data, then observe percentage).

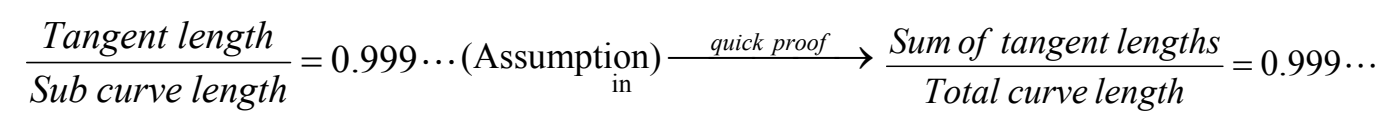

Infinite number of 9's will be added, as $0 . \dot{9}(=1)$ or numerator = denominator. Define:

Integral of tangent length $=$ Curve length

This is an extension of circle's circumference.

PS: Why is the curve length calculated based on tangent line rather than secant line? Our daily experience suggests to use pace to measure, i.e. the secant length step by step. When the pace becomes shorter and shorter, the secant length would become identical to the tangent length. The tangent length can be measured from the tangent point, without need to consider the other end point.

Figure 16. Tangential gaph.

Good news: The tangent can be defined as the differentiation in the next section (see Figure 16).

How to calculus the tangent length? We need to calculate the height, see the next section.

\section{The Third Plays: Calculate Height of Hillside}

No need to start all over again. Simply take the isomorphism and change one to the other (see Figure 17).

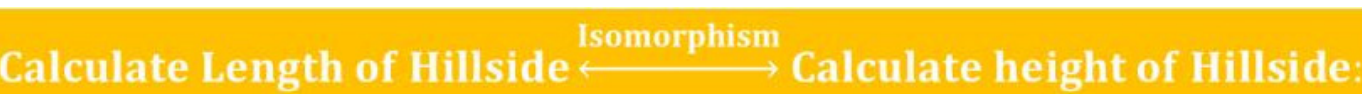
Convert the word "length" to "height"
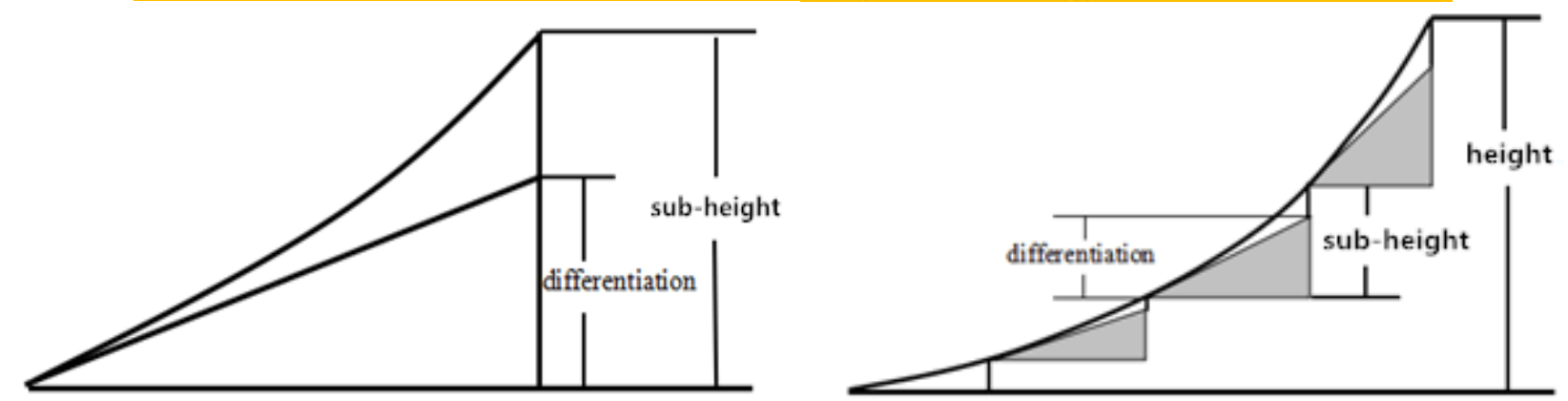

Figure 17. Sub-triangle and global triangle.

Percentage:

$$
\frac{\text { Differentiation }}{\text { Sub-height }}=0.999 \cdots(\text { Definition }) \stackrel{\text { quick proof }}{\longrightarrow} \frac{\text { Sum of differentiations }}{\text { Total height }}=0.999 \cdots
$$

in Lesson one 
Data Collection When the hillside is a parabolic curve:

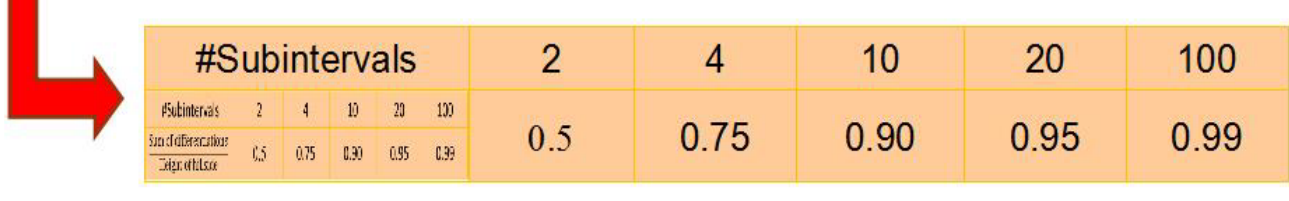

Figure 18. Data table.

The percentage also has one 9, two 9's, three 9's, four 9's ... (see Figure 18).

Infinite number of 9's will be added, as $0 . \dot{9}(=1)$ or numerator = denominator. Define:

$$
\text { Integral of differentiations }=\text { Height of hillside }
$$

Here, the integral is deterministic and finite. Good news: It could be exact value instead of approximate, thus solved the historical puzzle (e.g., circumstance). It is called the fundamental theorem in calculus textbooks, and here, it is called the common theorem.

\section{Conclusion}

Calculus, from hypothesis to theorem, is transferred to arithmetic level

$$
\begin{aligned}
& \text { Either } \quad 1>\frac{\text { differential }}{\text { sub-height }}>0.9 \ldots 9 \quad \text { or } \quad 1>\frac{\text { sub-height }}{\text { differential }}>0.9 \ldots 9 \\
& \Rightarrow \frac{1}{0.9 \cdots 9}>\frac{\text { sum of differentials }}{\text { sum of sub-heights }}>0.9 \cdots 9
\end{aligned}
$$

and then is proved by a few lines of arithmetic.

\section{References}

Jansson, J., \& Johnson, C. (2010). Body and soul. Search online.

Hua, L. G. (1981). Optimization method. Science Press.

Lin, Q. (1999). Calculus comics. Guangxi: Guangxi Normal University Press.

Lin, Q. (2013). Fast lane calculus (3rd ed.). Science Press.

Lin, Q. (2015). Data-based introduction to calculus. In Proceedings of The 20th Asian Technology Conference in Mathematics. Lin, Q., \& Yang, W. C. (2010). Making teaching calculus accessible. Electronic Journal of Mathematics and Technology, 15. Livshits, M. (2004). You could simplify calculus. Retrieved from http://arxiv. org/ps_cache/arx-iv/pdf/0905/0905. 3611vl. pdf Range, M. (2016). What is calculus? World Scientific Publishing Co. Pte. Ltd..

Shen, S., \& Lin, Q. (2014). Direct calculus simplifies teaching to 2-hours. Mathematic Teaching. (in Chinese)

Strang, G. (1991). Calculus. Wellesley, M.A.:Wellesley-Cambridge Press.

Terence, C.-S. T. (2008). Real analysis. Posts and Telecommunications Press.

Yang, W. C. (2012). Students will excel if they are inspired. Plenary talk at ATCM. Retrieved from http://atcm.mathandtech. org Zhang, J. (2012). Calculus without limit. Chinese Children Press. 


\section{Appendix: Pointwise Differentiation and Interval Differentiation}

For calculus teachers, who are familiar with the derivative definition.

We have had pointwise derivative and interval derivative. The latter is defined as: For all $x$ :

$$
\left|f(x+h)-f(x)-h f^{\prime}(x)\right|<\varepsilon h
$$

or $\mid$ sub-height-differentiation $\mid<\varepsilon \cdot$ base .

From Lin (1999), the definition of the interval derivative contains the Newton-Leibniz formula.

Correspondingly, there are the pointwise differentiation and the interval differentiation. The latter is defined as: For all points:

$$
\text { either } \quad 0.9 . .9<\frac{\text { differentiation }}{\text { sub-height }} \leq 1 \quad \text { or } \quad 0.9 \cdots 9<\frac{\text { sub-height }}{\text { differentiation }}<1
$$

where, differentiation $=$ tangent height $=f^{\prime}(x) h$. By taking inverse:

$$
\frac{\text { sub-height }}{\text { differentiation }} \geq 1>0.9 \ldots 9 \quad \text { and } \quad \frac{\text { differentiation }}{\text { sub-height }}>1>0.9 \cdots 9
$$

for each point simultaneously. Hence, their lower bounds are $0.9 \ldots 9$.

From the lemma in Lesson one:

$$
\text { either } 1>\frac{\text { sum of differentiations }}{\text { total height }}>0.9 \ldots 9 \text { or } 1>\frac{\text { total height }}{\text { sum of differentiations }}>0.9 \ldots 9
$$

and hence, the Newton-Leibniz formula.

Calculus teachers are familiar with the derivative concept rather than the differentiation concept.

Next, we will prove that the pointwise differentiation and the interval differentiation are just the same as the pointwise derivative and the interval derivative, respectively, under certain conditions. Therefore, if the definition of derivative is reasonable, so is the differentiation.

Proposition: If $f(x)$ has pointwise derivative and $f^{\prime}(x) \neq 0$, then $f(x)$ has pointwise differentiation: Take any number of 9 after the decimal point $(0.9 \ldots 9)$, we have, at each $x$, when the partition is small enough (depending on the point).

$$
\text { either } \quad 0.9 \cdots 9<\frac{\text { differentiation }}{\text { sub-height }} \leq 1 \quad \text { or } \quad 0.9 \cdots 9<\frac{\text { sub-height }}{\text { differentiation }}<1
$$

Proof: Since $f(x)$ has pointwise derivative, i.e., $\forall \boldsymbol{\varepsilon}>\mathbf{0}, \exists \boldsymbol{\delta}>\mathbf{0}$, when $0<h<\delta$ (where $\boldsymbol{\delta}$ depends on $x$ ):

$$
\left|\frac{f(x+h)-f(x)}{h}-f^{\prime}(x)\right|<\varepsilon \Rightarrow\left|\frac{f(x+h)-f(x)}{h f^{\prime}(x)}-1\right|<\frac{\varepsilon}{\left|f^{\prime}(x)\right|}=C(x) \varepsilon
$$




$$
\begin{aligned}
& \text { Hence, } 1-C(x) \varepsilon<\frac{f(x+h)-f(x)}{h f^{\prime}(x)}<1+C(x) \varepsilon \\
& \text { 1.If } \frac{\text { sub-height }}{\text { differentiation }}<1 \text {, then, } 1-C(x) \varepsilon<\frac{\text { sub-height }}{\text { differentiation }}<1 . \\
& \text { 2.If } \frac{\text { sub-height }}{\text { differentiation }} \geq 1 \text {, then, by taking inverse, } \\
& \frac{1+C(x) \varepsilon}{1+\frac{\text { differentiation }}{\text { sub-height }} \leq 1, \text { where } \frac{1}{1+C(x) \varepsilon}=1-\frac{C(x) \varepsilon}{1+C(x) \varepsilon}>1-C(x) \varepsilon, \text { hence }} \\
& 1-C(x) \varepsilon<\frac{\text { differentiation }}{\text { sub-height }} \leq 1
\end{aligned}
$$

Therefore, the pointwise derivative deducts the pointwise differentiation when $f^{\prime}(x) \neq 0$.

Case 1: If $f(x)$ has interval derivative on $[a, b]$ and that the derivative is strongly positive $0<c<f^{\prime}(x)<C<\infty$ then $f(x)$ has interval differentiation.

From the lemma in Lesson one, the interval differentiation contains the Newton-Leibniz formula.

$$
\int_{a}^{b} f^{\prime}(x) d x=f(b)-f(a)
$$

Case 2: If $f(x)$ has interval derivative on $[a, b]$ and that the derivative is not strongly positive but bounded.

$$
-M<f^{\prime}(x)<M
$$

Let us consider the new function:

$$
F(x)=f(x)+(M+1) x
$$

where, $0<1<F^{\prime}(x)<2 M+1$. Hence, $F(x)$ has interval differentiation and has the Newton-Leibniz formula holds:

$$
\int_{a}^{b}(f(x)+(M+1) x)^{\prime} d x=f(b)-f(a)+(M+1)(b-a)
$$

Since $\int_{a}^{b} f^{\prime}(x) d x=-\int_{a}^{b}[(M+1) x]^{\prime} d x+f(b)-f(a)+(M+1)(b-a)$, we have

$$
\int_{a}^{b} f^{\prime}(x) d x=f(b)-f(a)
$$

A direct proof of such a formula (Lin, 1999), where we used the absolute values and the trigonometrical inequalities. 
Final Remark. The circumference has a convex primary function:

$$
4 \int_{0}^{1} \sqrt{1+\left[f^{\prime}(x)\right]^{2}} d x=4 \int_{0}^{1} \sqrt{1+\frac{x^{2}}{1-x^{2}}} d x=4 \int_{0}^{1}(\arcsin x)^{\prime} d x
$$

The circular area has a concave primary function:

$$
4 \int_{0}^{1} f(x) d x=4 \int_{0}^{1} \sqrt{1-x^{2}} d x=4 \int_{0}^{1} \frac{1}{2}\left(\arcsin x+x \sqrt{1-x^{2}}\right)^{\prime} d x
$$

